\title{
VENTAJAS COMPETITIVAS Y DESARROLLO METROPOLITANO. EL CASO DEL SISTEMA MANUFACTURERO TURINÉS
}

\section{Sergio CONTI}

Dipartimento Interateneo Territorio. University of Turin.

\section{INTRODUCCIÓN.}

En este trabajo se pretende dar cuenta de las iniciativas más recientes de análisis y de puesta en marcha de una estrategia de cluster dirigida a la revitalización de un sistema productivo antiguo, el de la región metropolitana de Turín. Por tradición altamente especializado en la producción automovilística, en los últimos decenios este sistema ha vivido acontecimientos complejos y contradictorios que se han manifestado en una caída drástica de la ocupación, en una descentralización de consistentes funciones manufactureras, en la racionalización tecnológica y organizativa del tejido empresarial y por otro lado en significativas señales de diversificación funcional.

A lo largo de la disertación, tesis teórico-metodológicas, analíticas y políticas se enlazan inextricablemente tanto a nivel lógico como a nivel de procedimiento. De hecho, cualquier solución estratégica no puede separarse de los fundamentos cognoscitivos de la realidad observada (los cuales constituyen con razón el fundamento). A la vez, la definición de una hipótesis de intervención política (en este caso, a cluster-implementation strategy) depende forzosamente de la interpretación que se da a las dinámicas estructurales de la economía en los decenios que cierran el milenio.

Por estos motivos, el trabajo se dividirá en diferentes partes aunque presentadas de modo secuencial cuyo conjunto podrá explicar las hipótesis que han inspirado una estrategia de cluster-implementation y las metodologías adoptadas en los últimos años por las instituciones locales. En la primera, el problema de la ventaja competitiva regional y de las lógicas de interpretación implícitas se afirma como expresión de las dinámicas estructurales que afectan a la economía contemporánea, cuyos fundamentos se presentarán de nuevo aquí, aunque de forma muy esquemática.

En la segunda se examinarán las metodologías (cuantitativas y cualitativas) a través de las cuales se ha llegado a delinear la existencia de sistemas de empresa de tipo cluster que operan en la región de Turín, de cada uno de ellos se tenderá a definir las lógicas fundamentales de funcionamiento y las relaciones (reales y latentes) existentes en su interior. Más concretamente, el sistema manufacturero turinés ha sido subdividido en ocho cluster agrupados en tipologías diferentes: a)

Polígonos, nº 9, 1999, pp. 49-69. 
estratégicos, b) innovativos, c) tradicionales.

En la tercera parte se tratará de averiguar las lógicas de interpretación del sistema turinés y por consiguiente las estrategias de intervención política a partir de los cluster que se han puesto de relieve. En esta sección se dará cuenta del descubrimiento de una "estrella de la competitividad", fundada en cuatro estrategias de revitalización industrial diferentes y, al mismo tiempo, relacionadas: a) innovación tecnológica, b) formación profesional, c) comercialización, d) planificación urbanística. La lógica que contiene es la de una estrategia radial dirigida a la formación de relaciones de colaboración (además de competitivas) entre un amplio abanico de sujetos presentes en el sistema, con la intención de "superar" la condición de dominación de la producción automovilística, pero al mismo tiempo coherente con la herencia tecnológica y cultural que se deriva de ella.

Para concluir, en la última parte se examinarán los problemas de la puesta en marcha de una estrategia de política industrial, centrada en la creación de "Observatorios de cluster", la reorganización de las oficinas encargadas de la programación económica y urbanística locales, la institución de una Agencia especial para la política industrial.

\section{LA COMPETITIVIDAD REGIONAL EN LA ERA DE LA GLOBA- LIZACIÓN.}

Lo que más sorprende a quien observa los acontecimientos económicos de nuestro tiempo es que los fundamentos en base a los cuales habían vivido las economías desarrolladas en la posguerra, una época dorada, ya no existen. Hace ya dos decenios que las señales de recuperación económica -en el momento en el que se aprecian- se realizan en un cuadro de desempleo permanente, de salarios reales estancados, de desigualdades crecientes en los niveles de beneficio de las empresas. En otras palabras, durante los años 80 y 90 los indicadores macroeconómicos ya no reflejan la coherencia que había definido los caminos del desarrollo de la posguerra.

Existe una fuerte sensación de que se ha trazado una linea divisoria, está viva la percepción de la pérdida de viejas certezas. Con anterioridad hemos vivido años de confusión, pero, precisamente por esto, también de incesantes búsquedas de respuestas y soluciones nuevas. De todos modos, es verdad que en los últimos años algo ha aparecido en el horizonte y que nuevas posibilidades interpretativas se pueden percibir por fin.

Para entenderlo mejor, podemos examinar una secuencia rápida de pocos elementos clave. Estos no agotan claramente una problemática íntimamente compleja, así mismo cada uno de ellos necesitaría en realidad un tratamiento más amplio.

1. La creciente internacionalización de las actividades productivas produce el efecto de inducir una especie de tendencia a la ubicuidad de los factores de producción. La consecuencia, a primera vista, es la erosión de las capacidades productivas de muchas economías nacionales y regionales.

Esta es una antigua idea que los economistas aprendieron de los trabajos fundamentales sobre la "resource base theory" de E. PENROSE (1959), que quizá por 
primera vez divulgó la idea según la cual, cuando la ventaja competitiva se basa en capacidades escasas y heterogéneas, una economía regional o nacional "ganadora" tendrá que disponer de capacidades que otros no posean (y por tanto raras) y valorizables dentro de un escenario más amplio.

Si es verdad que en un mundo donde virtualmente todos saben hacer las mismas cosas en tiempos y con costes sustancialmente análogos no puede hablarse de ventaja competitiva, la historia contemporánea nos enseña que algunos recursos ubicuos en realidad no lo son. Es bastante fácil pensar en el trabajo, por ejemplo, que sigue siendo un factor sustancialmente inmóvil.

Sin embargo hay otro factor, cada vez más determinante en la economía contemporánea y también caracterizado por una elevada inmovilidad: se trata de la capacidad de producir conocimiento. Se sabe que muchas empresas que operan en países con costes elevados de trabajo, han respondido a la tendencial ubicuidad y a la relativa reducción del coste de los factores, generando rentas empresariales a través de la creación de conocimiento. Sobre todo en los países industrializados y con un alto coste de los factores de producción (del trabajo en primer lugar) el pasaje gradual hacia la economía del conocimiento se puede interpretar en primer lugar como resultado del tendencial proceso de globalización de los factores y de los mercados. En estas regiones, el problema de la competitividad se centra de manera creciente en la capacidad de crear, acumular y utilizar el conocimiento más rápidamente que los competidores. Es la creación de conocimiento (intencional, con trayectoria fuertemente subordinada a las prácticas y a las rutinas empresariales y locales) y no el conocimiento producido (transferible de forma codificada) la que representa el nuevo gran factor localizado, fuente de la ventaja competitiva en la economía globalizada contemporánea.

2. Es un hecho que el proceso de globalización va acompañado de un crecimiento bastante rápido de los flujos de inversiones internacionales y de los productos. A pesar de eso, limitarnos a esta afirmación no nos llevaría lejos y se daría una imagen reducida de la realidad.

Lo que más desconcierta quizá a los economistas que dedican su atención a la emergente economía mundial es, en cambio, el creciente índice de realización de productos diferentes por parte de regiones y países, a través de procesos e instrumentos organizativos bastantes diferentes también. Es una evidencia estadística el hecho de que la especialización internacional por productos ha crecido regularmente en los últimos lustros, sobre todo para las economías del mundo industrializado (un fenómeno a primera vista sorprendente en una época en la que la utilización de las comunicaciones y de la informática favorece como nunca hasta ahora la difusión y la imitación de la tecnología)

Esto significa que la creciente especialización de las economías (nacionales, regionales) ya no depende de las economías de escala en la producción -y por tanto de la relación competitividad/precio- sino de la naturaleza de los productos que se introducen en el mercado, de los "saber hacer" que realizan estos productos, del tipo de necesidades que estos satisfacen, de la capacidad de hacer evolucionar de modo continuo dichos productos, preservando sin embargo su originalidad.

3. Está claro que en estas condiciones la posibilidad de que una economía 
regional se proponga con éxito en los mercados internacionales se halla, por un lado en la identidad conferida al producto, o sea en lo que lo diferencia del de los competidores y, por otro, en el hecho de que la solución al problema de la ventaja no se encuentra ya en la búsqueda externa de las tecnologías y de los métodos de producción disponibles que se consideran mejores. Si existe una solución habrá que buscarla internamente en la misma región, o sea en la capacidad de coordinación de productores, consumidores, instituciones y otros actores localizados.

El desafío, en otras palabras, es de naturaleza organizativa e implica a los sujetos y a su lógica de acción y de comunicación. Se trata de un aspecto que se revela más importante en la medida en que la teoría económica tradicional ( $\mathrm{y}$ todavía dominante) se muestra incapaz de asumir la lógica de acción de los sujetos, no pudiendo comprender la misma si se separa la dimensión económica de las otras dimensiones, que son histórica y territorialmente específicas.

4. Lo que se ha sostenido hasta ahora posee una comprobación propia. La globalización (la formación de redes globales de empresa) debilita la soberanía económica de los Estados nacionales y refuerza por consiguiente la especialización regional en las actividades competitivas. A su vez, las regiones se suman a la economía global promoviendo las propias especializaciones. Esto explica la difusión de estrategias y decisiones políticas (a menudo neomercantilistas) dirigidas a la promoción y refuerzo de los sistemas (o cluster) presentes, o sea conjuntos de sujetos y actividades dialógicamente relacionados y por esto generadores del valor.

Estas consideraciones tendrían un sentido muy abstracto si no encontraran fuertes confirmaciones, que se obtienen observando las historias de éxito que han descompuesto en cierta medida el planisferio de nuestro mundo industrial. Limitando la observación a la experiencia de áreas con una antigua tradición manufacturera, se deduce que efectivamente numerosas regiones (en Europa, por ejemplo el País de Gales, la cuenca del Ruhr, Westfalia, Baden Wurttenberg, la región de Lyon, Cataluña, por citar los ejemplos más conocidos) en los últimos lustros han reciclado con éxito las propias estructuras productivas.

Se trata de casos muy diferentes, y eso bastaría para reforzar la tesis de lo injustificado que sería, en nuestro complejo mundo, volver a encontrar un "modelo" unitario para trasladarlo a otra parte. Sin embargo, es legítimo asumir algunas razones genéricas de este éxito que a nivel de método efectivamente pueden hacer que encontremos un rastro de esperanza. De nuevo limitémonos a una breve (y con toda seguridad incompleta) reseña.

a) Para despejar el campo de fáciles determinismos, lo primero que se deduce es que en ningún caso el reciclaje de las estructuras productivas ha sido realizado en un intento de atraer a las industrias convencionalmente definidas como industrias de alta tecnología (biotecnologías, semiconductores, aeronáutica, software, etc.). Esto no significa obviamente que no se haya perseguido una política tecnológica. Sin embargo, ésta (y este es el aspecto relevante) se ha dirigido a la utilización y a la "regeneración" de recursos tecnológicos históricamente radicados en la economía de la región.

b) El reciclaje de la economía de la región no ha tenido lugar con la promo- 
ción de nuevas improbables actividades (ver, por ej., HUDSON, 1995), sino persiguiendo el fomento de las actividades manufactureras. Si todas estas regiones ganadoras disponían ya de una estructura industrial integrada con diferentes grados de complejidad, las soluciones se han buscado en el marco del respeto (además del apoyo y de la regeneración) de estas realidades productivas, promoviendo a la vez la especialización y la diferenciación funcional (REHFELD, 1995). Dándole la vuelta al discurso se puede sostener que los casos con éxito han sido aquellos en los que estaba presente (o latente) una gama más o menos amplia de categorías de ocupación en un número limitado de sectores: en práctica una estructura en forma de cluster de la economía, o la presencia de sistemas productivos parciales en cuyo interior se llevan a cabo vínculos organizativos relativamente estables.

c) Por todas partes se han perseguido (o se han puesto en marcha) estrategias de red destinadas a favorecer y sostener la interacción de sujetos - entre empresas, entre empresas y otras instituciones, entre diferentes instituciones - a nivel de asistencia y apoyo financiero y tecnológico. La creación del llamado capital social (PUTNAM, 1993) va unido a formas de intervención para apoyar la formación de pequeñas empresas y la predisposición a condiciones de learning economy (LUNDVALL y JOHNSON, 1994), caracterizadas precisamente por la colaboración e interacción a nivel regional de empresas con the science base, pública o privada.

d) Todas estas regiones, para terminar, disponían de instituciones relativamente fuertes e intervencionistas, capaces de activar la formación de grupos de interés que no se limitan a perseguir objetivos y conocimientos específicos sino que disponen de un horizonte normativo común, una agenda política compartida (HAAS, 1992). Es obvio que esta institutional tickness se realiza con más facilidad a nivel regional (y metropolitano), siendo esta la escala más adecuada para incentivar la formación de relaciones más sólidas entre los sujetos presentes, para la realización de estrategias de coordinación, para la movilización de los recursos presentes. En otras palabras, esos casos ganadores presuponen una especie de "regionalización de la política regional".

Resumiendo, si se puede extraer una conclusión de lo recordado hasta ahora, es que la regeneración económica:

- Por un lado, no se ha reducido a un set de restringidos factores económicos (que son esenciales de todos modos), sino que ha implicado con fuerza a factores institucionales, culturales y sociales. Eso halla por otra parte sólidos fundamentos teóricos tanto en la "nueva sociología institucional" con su énfasis en la radicación (GRANOVETTER, 1985; HODGSON, 1993), como en las tesis de la escuela neoschumpeteriana, según la cual la innovación, en cuanto fenómeno evolutivo, yace en un proceso interactivo y toma forma en las rutinas institucionales y en las convenciones sociales (DOERINGER y TERKLA, 1990; DOSI, 1998; FREEMAN, 1994; LUNDVALL, 1992);

- Por otro lado, el hecho de que se debe transcender cualquier hipótesis de política industrial "genérica" para privilegiar, por el contrario, soluciones estratégicas selectivas, dirigidas precisamente a la persecución del refuerzo de la cooperación interindustrial dentro de sistemas de sujetos (en sentido lato, clus- 
ter) que conjuntamente poseen capacidades valorizables a nivel internacional, obteniendo un beneficio (de nuevo, conjuntamente) de las condiciones ambientales existentes o producibles. A nivel de método esto marca el pasaje de la política sectorial a la política por sistemas, como instrumento que permite establecer mejor los correspondientes puntos de fuerza y de debilidad de la estructura productiva y a partir de estos prefigurar los instrumentos de intervención capaces de valorizar al máximo los efectos externos de la co-localización (COOKE y MORGAN, 1993; FOSS, 1996).

\section{EL RECORRIDO EMPÍRICO Y LOS CLUSTER REALES.}

Está extensamente codificada la imagen de Turín como company town, sede histórica de la industria automovilística italiana y uno de los centros mayormente especializados e industrializados de Europa. Al mismo tiempo, Turín ha contituído quizá la expresión más típica, en este siglo, de las lógicas organizativas y sociales ford-tayloristicas, que han dado protagonismo a la mayor empresa italiana privada, la Fiat.

La historia industrial reciente ya se ha encargado de explicar las profundas transformaciones que han tenido lugar en el sistema turinés a partir de la enérgica introducción de innovación, tanto en campo tecnológico como organizativo (CONTI and ENRIETTI, 1995; VOLPATTO, 1966). En las últimas dos décadas la realidad productiva y social de la ciudad ha sufrido significativas transformaciones, que en una síntesis extrema podemos ajustar a tres valores fundamentales:

- Antes que nada, las economías de amplio espectro han ido sustituyendo a las economías de escala en la determinación y consolidación de las claves del éxito productivo;

- Contemporáneamente, las rutinas organizativas de las empresas turinesas, tanto de grande como de medio-pequeña dimensión, han estado en la base de la consolidación de un sistema estratégico de creación del valor que es expresión explícita de la radicada producción automovilística. Este comprende, junto al sistema de empresas abastecedoras de componentes para vehículos, un sólido tejido de proyectistas y car designer y un conjunto relevante de productores de máquinas herramienta, con resultados excelentes en el campo de la robótica y de la automatización industrial;

- Para terminar, la masiva introducción de innovaciones de proceso ha llevado a numerosas empresas tradicionales a dirigirse hacia combinaciones productivas más eficientes, sin comprometer por esto la trama de transacciones y de relaciones de "lealtad" con la gran empresa dominante.

Pero en el ápice de estos procesos existe una consolidada opinión de que los hechos confirman los caracteres estructurales de la economía turinesa: el sistema se caracteriza todavía por una estructura empresarial e industrial fuertemente "integrada" y "concentrada", aunque se revelen algunas tendencias a una creciente diversificación sectorial.

Estas breves consideraciones hablan claro sobre el hecho de que la realidad es mucho más compleja de lo que podría resultar a primera vista asumiendo los indicadores estadísticos habituales. Y es así para las políticas: las opciones con- 
vencionales ya no ofrecen soluciones creíbles a medio y largo plazo. Al contrario, se requieren soluciones estratégicas innovativas.

El problema es revalorizar y dar un sentido completo a la dimensión estratégico-subjetiva de la empresa y a los procesos localizados de interacción entre empresa y ambiente. De este modo, y dentro del respeto de las opciones de método expuestas con anterioridad, las condiciones ambientales geográficamente específicas y no repetibles se convierten en protagonistas de la reflexión sobre la competitividad regional en la era de la globalización de las elecciones económicas.

Es desde este punto de vista que el concepto de cluster (entendiendo con él la parte espacialmente concentrada de una red productiva, al mismo tiempo relacionada con los mercados globales y fundada en un potencial endógeno de valorización) parece desde el principio el instrumento - teórico y programático- capaz de expresar la transición del análisis de sector (y de empresa) precisamente al de sistema.

En el momento del pasaje a la discusión empírica, esta dialéctica sector/sistema asume significados en los que se compenetran tanto instancias teóricas como objetivos de procedimiento. Más en concreto, esta esconde la misma dialéctica entre métodos cuantitativos y métodos cualitativos, con la consecuencia de haber orientado los trabajos de investigación a lo largo de dos direcciones estrechamente relacionadas entre ellas:

- Por un lado, el análisis cuantitativo, dirigido a establecer sectores dinámicos presentes en la región turinesa, como presupuesto para la identificación de los cluster manufactureros;

- Por otro lado, el análisis cualitativo, dirigido a la comprensión plena del significado y el funcionamiento de los cluster establecidos. En esta segunda fase, la introducción del concepto de sistema del valor, en el que se han introducido los valores y las funciones organizativas propias del ambiente, permite trascender las meras relaciones funcionales y tecnológicas, introduciendo en el análisis aquellos elementos complejos que no se perciben en el nivel precedente.

Por tanto sobre esta base es posible dividir los trabajos de investigación llevados a cabo por el team ${ }^{1}$ en dos fases: la primera, con bases cuantitativas, de tipo macroscópico y la segunda, con bases cualitativas, de tipo microscópico.

\section{a) El análisis macroscópico.}

Desde el punto de vista del método utilizado, este se articula en tres momentos principales.

a.1. La elección de los datos. El análisis se ha basado en los datos del censo de la industria llevado a cabo por el ISTAT en 1981 y en 1991 y en las bases de datos de demografía industrial proporcionadas por el Instituto Nacional de la Seguridad Social (INPS). Esto ha permitido trabajar sobre todo el conjunto de empresas presentes en el sistema local turinés, y no sobre una parte sólo.

\footnotetext{
${ }^{1}$ El grupo de trabajo, instituido por el Ayuntamiento de Turín y coordinado por Sergio Conti, ha implicado a la local CCIAA, a la Unión Industrial, a la Diputación provincial de Turín, a la Agencia para la Promoción de Turín y de Piamonte, además de contar con la colaboración de centros de investigación universitarios y privados.
} 
a.2. El área de investigación. Desde el punto de vista de su disgregación espacial, el área observada se ha dividido del siguiente modo: el ayuntamiento de Turín, la primera área metropolitana (que comprende 41 ayuntamientos), la segunda área metropolitana (24 ayuntamientos).

En particular, por lo que se refiere a la definición de primera área metropolitana, se ha elegido el criterio de "área funcional" como la ha definido recientemente el ISTAT-IRPET (1994). En concreto, se establece como sistema elemental el lugar donde se desarrolla la mayor parte de la vida cotidiana de la población, o sea el mercado local del trabajo dentro del cual se llevan a cabo los intercambios de bienes, información y servicios entre individuos y grupos humanos, que comprende también las sedes en las que tales actividades se desarrollan.

a.3. Articulación e instrumentos de análisis. Concretamente, el análisis se ha basado en un conjunto de indicadores estadísticos, desde los más sencillos (como las variaciones porcentuales) a otros de naturaleza más compleja, como los coeficientes de localización y el análisis shift-share.

A partir del análisis realizado de los distintos sectores de la manufactura turinesa aparecen 9 sectores particularmente dinámicos:

- Instalaciones para el control de los procesos industriales

- Máquinas para usos especiales

- Máquinas para oficina

- Tubos y válvulas electrónicas

- Partes y componentes para automóviles

- Aeromotores y vehículos espaciales

- Prensa y servicios relacionados

- Artículos de papel y cartón

- Aparatos para telecomunicaciones

En una segunda fase, utilizando la base de datos del INPS, se han añadido a estos sectores dinámicos los sectores relacionados con ellos (o sea, que forman parte de las mismas categorías de mercancía o que presuponen de todos modos unos vínculos funcionales más o menos intensos), llegando al establecimiento de cinco grupos de actividad que constituyen, de hecho, una primera estructura de algunos cluster: 1) producción de vehículos, 2) producción de máquinas herramienta, 3) telecomunicaciones, 4) espacio aéreo, 5) prensa y actividad editorial.

\section{b) El análisis cualitativo.}

Los cluster manufactureros que se han establecido "estadísticamente", aunque representan una base de apoyo imprescindible para llegar a la representación de las dinámicas estructurales del sistema, no podían no dejar sin resolver demasiadas ambigüedades. Por este motivo se ha hecho necesario desarrollar un conjunto de instrumentos cualitativos de análisis que permitan gestionar la complejidad de un sistema local como el turinés. Se trataba, en otras palabras, de ir a la raíz de los sistemas puestos de relieve con anterioridad, integrando el análisis estadístico con estudios directos, con el fin de llegar al núcleo de las modalidades de funcionamiento, de la naturaleza, la intensidad y la evolución de las relaciones tecnológicas y funcionales que tienen lugar en su interior.

El análisis ha seguido un camino articulable en tres fases diferentes, pero 
lógicamente relacionadas:

b.1. Recogida de los elementos fundamentales para conocer el funcionamiento de cada uno de los cluster puestos de relieve. Para este fin el grupo de trabajo se ha valido de la colaboración de algunos observadores privilegiados (especialistas de sector, que operan tanto en la Universidad como en otros centros de investigación de la región y exponentes de relieve de empresas clave presentes en el área de Turín) capaces de orientar los conocimientos de forma coherente hacia los fines del estudio. Se trataba de un pasaje necesario para dar un significado a los "nudos" (las empresas) que componen el sistema, por lo que el establecimiento de los cluster (o sistemas productivos parciales) ha ido surgiendo en el curso de los trabajos de observación y ha derivado de la toma en consideración de aquellos segmentos del sistema en los que se tejen vínculos organizativoterritorializados relativamente estables.

b.2. Realización del cuestionario. La selección de las empresas (unas 200 en total) a las que había que dirigir el cuestionario se ha basado:

- En la exigencia de profundizar el conocimiento de cada "nudo" que compone el cluster que se analiza;

- En las indicaciones proporcionadas por los observadores privilegiados, y por tanto

- En el papel estratégico que juegan algunas empresas en el funcionamiento general del sistema.

- En la información que se deriva, paso a paso, de la realización de los mismos cuestionarios.

Para marcar en mayor medida el alejamiento de las metodologías que caracterizan la primera parte de la investigación, la selección analizada no es entonces de naturaleza estocástica sino que emerge de una lectura del universo manufacturero llevada a cabo a través de filtros cualitativos. Un camino de este tipo presuponía que la selección no se definiera a priori sino que quedara abierta para recoger modificaciones e incorporaciones a medida que procediera la investigación directa evidenciando, por ejemplo, nuevas relaciones de colaboración y de competencia en el interior del cluster o entre diferentes cluster. Está claro que actuando de este modo se asume que virtualmente el análisis no tiene final: añadiendo observaciones, la fenomenología estudiada se hace cada vez más inteligible, mientras que los resultados obtenidos precedentemente se modifican y se ponen en tela de juicio constantemente.

b.3. La organización de la información adquirida ha seguido, a su vez, dos lógicas precisas, que se pueden sintetizar del siguiente modo:

i) Se han establecido los caracteres estructurales, la dinámica, los "puntos fuertes y débiles" de cada cluster y, por consiguiente, del sistema manufacturero turinés en su conjunto. Esto ha permitido resolver interrogantes fundamentales sobre la consistencia (y la radicación) de algunas actividades del territorio turinés, por un lado, y por otro, sobre su capacidad competitiva;

ii) Se han establecido los recursos localizados o producibles, las condiciones de funcionamiento y las exigencias de efectos externos específicas para cada cluster. En este sentido, ha constituido una referencia esencial el análisis de las condiciones de localización esperadas, presentes y latentes y por esto potencial- 
mente funcionales como soporte de su dinámica competitiva.

Por tanto el pasaje de los cluster estadísticamente relevantes a la representación de los cluster "reales" ha llevado a modificar y enjuiciar sensiblemente los resultados que han emergido de la primera fase de los trabajos. Los ocho cluster reales sobre los que se basa la economía manufacturera turinesa, según las diferentes dimensiones, características de estructura y dinámicas competitivas, se pueden escindir en tres clases:

a. Estratégicos, cuyos determinantes de competitividad están básicamente localizados en el área turinesa y constituyen al mismo tiempo el eje portante de la economía metropolitana (Máquinas herramienta, robótica y automatización industrial; Vehículos; Diseño y proyección; Telecomunicaciones);

b. Innovativos, cuyos determinantes de competitividad son de nuevo expresión del milieu metropolitano, aunque se caracterizan por relaciones intersistémicas menos fuertes que las precedentes (Sistemas de alarma; Vehículos espaciales);

c. Tradicionales, en los que se diluye el efecto-milieu de frente a condiciones ocupacionales, tecnológicas y de competencia menos pronunciadas, o deben su radicación a razones fundamentalmente históricas (Artes gráficas y actividad editorial; Bolígrafos).

A estos se suman dos amplios y heterogéneos sistemas manufactureros de soporte, en parte expresión de la historia manufacturera de la región, en parte inducidos por la dinámica tecnológica y competitiva de los sistemas que sostienen la economía. En gran medida se representan como "nebulosas" de empresas de pequeña y mediana dimensión.

Este modo de actuar ha permitido volver a leer e interpretar la economía manufacturera turinesa sacando a la luz diferentes dimensiones en las que se basa la competitividad. 1. La intensidad de las relaciones entre los diferentes componentes de cada cluster, 2. la consistencia y la radicación de algunas actividades específicas del territorio turinés, 3 . su capacidad competitiva. Gracias a esto ha sido posible hacer inteligible la estructuración y los problemas de los diferentes cluster, sacando a la luz, por ejemplo los eslabones débiles o perdidos de su organización o, viceversa, los elementos fuertes. Esto es plenamente coherente con los criterios que inspiran el proyecto de trabajo, que se fundan precisamente en el establecimiento de los sistemas de creación del valor hacia los que hay que dirigir una estrategia no banal (y no indiferenciada) de política industrial.

b.4. Sistemas de valor y políticas de red.

Esta modalidad de razonamiento destruye irremediablemente muchas categorías consolidadas que han dominado durante mucho tiempo los fundamentos y las políticas de desarrollo regional.

Ya se sabe que los principios tradicionales del desarrollo regional han entrado desde hace tiempo en una crisis irreversible. Nos basta con prestar atención al hecho de que en Europa las estrategias comunitarias más recientes se centran en dos conceptos nuevos y cruciales, que se asumen (y se hacen oficiales) conjuntamente: el de competitividad y el de cohesión (CEC, 1994).

Si durante décadas la política comunitaria se ha dirigido en mayor medida a la atención a los síntomas de los problemas regionales (como el paro, por ej.) y 
no a las causas (como los bajos potenciales de innovación) (DUNFORD, 1994; DE VET, 1993), las estrategias más recientes tienden a actuar lógicas reticulares (innovation networking y social capital) reconociendo oficialmente la necesidad de relacionar las estrategias supply side con las condiciones locales demand side. Admitiendo los errores del pasado (cuando el conjunto de subsidios para las regiones terminaba privilegiando las políticas de infraestructuras) las best practice guidelines más recientes están destinadas a (MORGAN, 1997):

i) Estimular los procesos de aprendizaje colectivo;

ii) Predisponer un stock suficiente de capital social;

iii) Combatir la inercia institucional.

Se reconoce hoy en día que las claves del éxito dentro de la economía global, que se pueden resumir con el concepto clave de desarrollo local, se hallan en medida creciente en factores intangibles (se reconoce que el aprendizaje tecnológico y organizativo es la expresión de valores, convenciones, conocimiento tácito) (ASHEIM y ISAKSEN, 1997; DOERINGER y TERKLA, 1990). Esta idea conocida no se encuentra únicamente en los programas comunitarios o en los esquemas lógicos elegantes de la "nueva" ética del desarrollo regional. Ante la crisis de las estructuras fordistas y keynesianas, la capacidad innovativa ya no se personifica en las maquinarias, sino que es el resultado de efectos externos positivos y de aprendizajes colectivos y localizados que trascienden las capacidades técnicas individuales (o de empresa). Estas nuevas lógicas de política de desarrollo regional, basadas en la construcción y la consolidación de relaciones con forma de red dentro de un definido framework institucional, se afirman inexorablemente en el mundo real de la producción.

En los análisis que se discuten aquí, que centran su atención en los actores y en su capacidad de proyectar y gestionar nuevos instrumentos de intervención, salta a la vista inmediatamente que de hecho está en curso una profunda transición, precisamente desde el punto de vista de las expresas exigencias de los actores, de una política "funcional" - que toma normalmente la forma de un apoyo indiferenciado tanto desde punto de vista económico como infraestructural, con una atención prevalente a la solución de problemas logísticos de empresa-a una política de red, que hace prioritario el apoyo a las relaciones de colaboración (además de las competitivas) entre sujetos.

Si especialización y diferenciación funcional, como se decía anteriormente, son las bases de la competitividad del sistema, el horizonte posible (y auspiciable) ya no se encuentra en la invención de nuevas improbables especializaciones sino en la diferenciación funcional -que las cualifique- de las que poseen sus raíces en la historia y la cultura turinesas, o sea en la identidad de los productos y de los sujetos que los realizan.

Según esto, la transición hacia nuevos instrumentos políticos basados en una estrategia sistemática de cooperación es una exigencia explícita expresada por aquellos cluster que tienen el aprendizaje (localizado) como fundamento de la competitividad: esto afecta a los cluster que ya están sólidamente establecidos en el sistema económico local (como Diseño y Proyección, por una parte y Máquinas herramienta, robótica y automatización industrial por otra) y también a aquellos para los que existen las condiciones, potencialidades y las primeras, aunque 
débiles señales de su afirmación (como el cluster Sistemas de alarma).

El cluster Vehículos (al igual que los cluster más tradicionales) no forma parte de los cluster para los que la búsqueda de la ventaja competitiva depende de la formación de sistemas de apoyo a las empresas y de la activación de las condiciones territorializadas (económicas e institucionales). Este hecho refleja claramente la imagen de un rico castillo que, sin embargo, la historia de las relaciones entre empresa y ciudad ha rodeado de altísimas murallas, despojando al sistema de empresas turinesas de una cultura cooperativa.

\section{LA "ESTRELLA" DE LA COMPETITIVIDAD.}

Pero ¿cuáles son sustancialmente las condiciones territorializadas (en parte comunes, en parte específicas de cada cluster) de cuya activación sería legítimo esperarse un incremento de la capacidad competitiva del sistema?

A este nivel el discurso no puede prescindir de un elevado grado de abstracción, puesto que el nexo que une a las líneas-guía derivadas del análisis con una posible puesta en marcha de estrategias de política industrial es demasiado incierto y depende del juego a menudo perverso en el que se mueven las estructuras institucionales, las culturas de empresa y los procesos de toma de decisiones.

Con el fin de llegar rápidamente a una conclusión, con el concepto de estrella de la competitividad del sistema turinés, se quieren sintetizar numerosos razonamientos sobreentendidos. En ella se han tratado de conjugar:

a) Las condiciones (o relaciones) activas que han determinado, tanto en la actualidad como históricamente, la radicación de los sujetos de cada cluster en el sistema turinés, que resultan al mismo tiempo las bases de las competitividad;

b) Las condiciones (o relaciones) latentes, o nunca llevadas a cabo o explotadas sólo en parte, pero cuya activación - que otras condiciones presentes en el sistema manufacturero turinés han hecho posible - favorecería de forma verosímil un refuerzo de las condiciones de competitividad (ya sea promoviendo formas de diferenciación funcional o reforzando, cualificándolas, las especializaciones propias del sistema).

Concretamente, se han establecido cuatro grandes determinantes de la competitividad, expresión a un tiempo de las relaciones establecidas ahora y de las posibles opciones de política industrial y de planificación: a) logística externa de empresa, b) formación, c) comercialización, d) innovación.

Resulta obvio que los diferentes cluster presentan grados y formas de radicación distintos con respecto a las condiciones presentes y/o latentes en el sistema, las cuales se traducen en hipótesis de política industrial diferentes y específicas. En la disertación siguiente se ha recogido además el variado universo de empresas (con frecuencia de pequeña dimensión) electrónicas, mecánicas y de moldeado, que constituye la mayor riqueza de la manufactura local, en gran medida sostén de los cluster dominantes y por este motivo escasamente capaz de llevar a cabo de forma autónoma aquellos procesos de diferenciación y autonomización que le permitirían superar las turbulencias del ciclo económico contemporáneo.

a) El primero, por tanto, tiene como objetivo la logística de empresa. Se trata del aspecto más fácilmente perceptible y bajo muchos puntos de vista menos 
problemático, ya que está relacionado con una lógica organizativa de matriz funcional, propia de una forma de concebir el espacio de modo indistinto, que se puede plasmar en relación con las exigencias de la producción.

En la actualidad las aspectos externos funcionales en sentido lato son explotados adecuadamente por los cluster Máquinas herramienta, robótica y automatización industrial, Bolígrafos y Sistemas de alarma (por eso aparecen como componentes activos de la competitividad). Por el contrario, las exigencias de racionalización parecen urgentes para el cluster Vehículos (la selección de abastecedores de componentes, su expansión dimensional y el nuevo posicionamiento estratégico y financiero que se deriva todavía esperan racionalizaciones adecuadas en sentido logístico) y el compartimento de las Artes gráficas, representado por un amplio abanico de empresas de pequeña dimensión cuyo funcionamiento se ve obstaculizado todavía por las carencias relacionadas con el posible acceso y la posible expansión dimensional y nueva localización fuera de la zona central y semi-central de la ciudad.

Para terminar, por lo que se refiere al cluster Diseño y proyecto, se va afirmando una exigencia, la de favorecer la "difusión concentrada" de las empresas en el interior del perímetro urbano, tanto para obtener ventajas de imagen, como para retener competencias muy arraigadas en Turín.

b) La formación y la calidad de la fuerza de trabajo constituyen un factor que determina la radicación en el sistema de algunos cluster muy especializados (Diseño y proyecto, Sistemas de alarma, Espacio aéreo, ciertos segmentos del cluster Máquinas herramienta, robótica y automatización industrial). Al mismo tiempo, la formación en gran medida todavía depende de los recursos de la empresa y esto afecta paradójicamente a sectores que por tradición sostienen el sistema manufacturero turinés (determinadas especializaciones mecánicas de la industria vehiculística y de las Máquinas herramienta y las Artes gráficas) y a otros mucho más innovativos (como la proyección a través de $\mathrm{CAD}$ ).

De eso resulta que una política de formación profesional y de dirección deberá responder prioritariamente a la exigencia de una especialización superior, a través de la participación de las empresas en los procesos de formación y de la intensificación de las relaciones entre el mundo de la producción y el mundo universitario. Las relaciones entre las empresas y las estructuras universitarias de la ciudad todavía están "institucionalizadas" sólo en el caso de un número relativamente reducido de empresas de mediana y gran dimensión, resultando por lo general ocasionales para las pequeñas empresas (OCDE, 1991).

Sin embargo formas de formación profesional "implícita", que se proponen facilitar la radicación y la reproducción de la capacidad, de los "saber hacer" tradicionales y/o innovativos, desde el diseño a la electrónica, desde las artes gráficas a la mecánica, pueden encontrar una importante valorización dentro de algunos proyectos específicos: desde el reconocimiento de la formación efectuada en el interior de los talleres mecánicos a la creación de atelier de diseño que, una vez abandonada la hiperespecialización que está atravesando el cluster, restablezcan las técnicas "de taller" (desde el desarrollo de la capacidad manual a la relación personal entre maestro y alumnos) que han formado a los grandes diseñadores turineses. 
c) Por lo que respecta a la capacidad de comercializar los propios productos en los mercados internacionales, esta aparece desarrollada sobre todo por las empresas que pertenecen al cluster Máquinas herramienta, robótica y automatización industrial (de hecho la exportación cubre más del $50 \%$ del volumen de negocios en alrededor de la mitad de las empresas) y por el car design, del que las empresas turinesas poseen de hecho el monopolio mundial. Análogas consideraciones afectan a los cluster de segmento, como los Sistemas de alarma y los Bolígrafos, en los que los productores turineses de mayores dimensiones consiguen consolidar posiciones de prestigio en un mercado internacional extremadamente competitivo.

Teniendo en cuenta que la participación internacional de los abastecedores de componentes automovilísticos depende todavía en gran medida de decisiones estratégicas del productor final, desde el punto de vista de una estrategia de política comercial más amplia, una acción de apoyo concertada podría orientarse de modo verosímil hacia dos direcciones principales:

i) La adquisición de pedidos internacionales. Obtendría beneficio de esto el tejido de pequeñas y medianas empresas que operan en los distintos sectores de la mecánica, que de este modo reducirían su dependencia de las dinámicas económicas y manufactureras locales. Se trata, en concreto, de estrechar más los vínculos de colaboración entre los car designer y sectores especiales del humus (en concreto el proyecto de moldes), de tal modo que se pueda explotar la atracción que ejercen los primeros en todos los mayores productores de vehículos (por ejemplo, organizando visitas guiadas y encuentros entre productores turineses y potenciales clientes extranjeros);

ii) Servicios de asistencia al desarrollo del producto. Esta es la parte más innovadora de la política de apoyo a la comercialización, orientada a la diferenciación funcional de la producción turinesa, favoreciendo de este modo la formación de potenciales segmentos competitivos a nivel internacional. Concretamente se trataría de identificar a los proyectos con nuevas líneas de producto que merecen ser desarrolladas (es fundamental en esta fase la colaboración de los car designer), de evaluar si son factibles y comercializables, de seleccionar (una vez más en el humus industrial turinés) los actores más idóneos para realizar la producción.

d) La innovación es el aspecto más problemático. El riesgo principal relacionado con la definición de políticas innovation-oriented es la proliferación de escenarios utópicos que no tienen en cuenta las peculiaridades del sistema manufacturero local. De hecho, no es un problema preparar intervenciones estándar que introducir en el territorio, sino potenciar el ambiente innovativo proprio de cada cluster, como emerge de la interacción de numerosos factores.

Teniendo en cuenta la realidad profunda del sistema turinés, aparecen dos líneas principales de política de innovación:

i) Investigación de materiales. Constituye una de las principales carencias del ambiente innovativo turinés, frente a una tradición arraigada de elaboración de los materiales (véase sobre todo la mecánica de precisión y el moldeado). Las posibles soluciones conciernen, a este respecto, al acceso al conocimiento, o sea poner a disposición las innovaciones desarrolladas fuera del sistema local turinés 
(mediante los instrumentos propios de los observadores internacionales de las tecnologías) y a la investigación de materiales alternativos. En este último caso, la innovación de los materiales debería acompañarse de una adecuada predisposición de servicios de asistencia y de la aplicación de los resultados de la investigación inmediata y continuada.

ii) Innovación electrónica. No se trata obviamente del formal obsequio a todos los que querrían multiplicar artificialmente los polos tecnológicos e informáticos, sino de aprovechar algunas peculiaridades del sistema turinés y, en concreto, de impedir que se pierda un conocimiento electrónico, único en Italia, hace tiempo arraigado sólidamente en la zona que gravita en torno a Ivrea. Por razones relacionadas con las dinámicas de los mercados internacionales, las soluciones no podrán afectar a la investigación de base ni a la componentística electrónica, donde la posición de grandes competidores internacionales parece hoy en día inatacable; sino que, al contrario, tendrán que dirigirse hacia uno o varios campos de la investigación aplicada en los que se prefiguren oportunidades de éxito. Entre las posibles direcciones se señala el campo de aplicaciones electrónicas e informáticas de la building automatition, la construcción automatizada, que implicaría con fuerza a algunos componentes valiosos de la manufactura turinesa: los cluster de las telecomunicaciones y de los Sistemas de alarma, además del sector de la automatización industrial.

El problema de prefigurar posibles intervenciones proyectivas que respondan a las instancias que se han recordado ahora, se concreta, como veremos mejor enseguida, en dos proyectos de planificación diferentes (que, por lo demás, poseen una colocación explícita en la figura).

El primero copia la lógica tradicional de parque tecnológico, en cuyo interior se lleva a cabo la circulación de la información y el refuerzo de la competitividad a través de la predisposición de servicios complejos y de asistencia a las empresas. Este se centraría en tres actividades principales:

- Investigación de materiales. O sea que el parque tendería a atraer a taylor maker y compounder, o empresas especializadas en la realización de materias "personalizadas" para aplicaciones de segmento;

- Sensorística y building automation. Indicada como una de las aplicaciones de la electrónica que gozará en el futuro de mayor desarrollo, la sensorística es susceptible de numerosas aplicaciones de segmento;

- Vehículos espaciales. La presencia de empresas que operan en el cluster Vehículos espaciales hallaría el refuerzo de la base electrónica y se abriría la posibilidad de ampliar el abanico de las producciones locales, sustituyendo componentes hasta hoy importados.

El segundo tiene por objeto el Parque generalizado del diseño y la creativi$\mathrm{dad}$. En este caso se trata de preparar políticas de red que faciliten la relación y, al mismo tiempo, la radicación territorial de las actividades más relacionadas con la "creatividad industrial" que, por su misma naturaleza, no pueden concentrarse en un parque tecnológico de tipo tradicional. Para estas actividades se puede actuar una política de concentración en un área urbana que haya que reciclar desde el punto de vista productivo, consintiendo a estas actividades (por lo demás no contaminantes y consumidoras de escaso espacio) su integración en el tejido so- 
cial de la zona.

Las actividades de las que se trata se pueden agrupar en las siguientes tres categorías:

- Industrial designer, car designer medianos y pequeños y actividades relacionadas (diseño, modelización, prototipización, etc.);

- Diseño y realización de moldes (excluyendo la actividad de moldeado de los materiales);

- Diseño y masterización de circuitos impresos y, en general, servicios electrónicos e informáticos.

La Administración Pública no puede no acompañarlas de la preparación de centros de formación, divisibles a su vez en dos categorías principales: escuelas para diseñadores $C A D$ (con una formación más especializada con respecto a los cluster turineses y en particular a Vehículos y Máquinas herramienta) y atelier, o sea, estructuras encomendadas directamente a diseñadores turineses. El mismo operador público intervendría en la preparación de un centro de apoyo al producto, o sea una estructura encargada de identificar proyectos y nuevas líneas de producto que merecen desarrollarse ${ }^{2}$.

\section{UN DESAFÍO INSTITUCIONAL.}

La aproximación en términos de cluster y de sistemas de valor ha permitido deducir las fundamentales ventajas competitivas que el sistema turinés presenta debido a la propia herencia histórica, a los procesos que, en los años recientes, han marcado las transformaciones del tejido manufacturero y a las "nuevas" condiciones competitivas.

Por consiguiente, una política que se propone influir en las condiciones de competitividad del sistema manufacturero turinés, deberá actuar con prioridad sobre algunas variables críticas selectivas dentro de un marco temporal a medio plazo. Esto implica una política industrial que opere a nivel de sistema y que "comparta" las tendencias en curso: la misma se define en términos de política de red, que se propone incrementar la cohesión y la radicación de los factores de la competitividad. De hecho, el desafío no es preparar servicios o estructuras "genéricas", sino crear específicas condiciones operativas, definiendo las bases fundamentales para el desarrollo de peticiones por parte de los sistemas más dinámicos.

Teniendo en cuenta los límites de acción de las administraciones públicas, el desafío es esencialmente organizativo, o sea que haga participar a los actores públicos como sujetos activos (y propositivos) en el desarrollo de un marco institucional coherente con las lógicas del sistema productivo, que se proponga eliminar los vínculos que inhiben la afirmación y aportar las intervenciones capaces de valorizar recursos latentes.

Esta aproximación está implícita en las hipótesis que han inspirado la investigación y, en concreto:

\footnotetext{
${ }^{2}$ Una estructura análoga la ha creado en Westfalia Dieter Siegel, fundador de la Siegel Design Consulting, con sede en el castillo de Harkotten.
} 
a) En la exigencia de que las instituciones (además de las empresas) se sensibilicen sobre las ventajas que derivan de la realización de una política no genéri$c a$ de apoyo a las relaciones entre los sujetos;

b) El análisis de la red de relaciones externas a las empresas, que se ha basado en el establecimiento de los nudos portantes de cada sistema;

c) El análisis de la potencialidad de red. En el recorrido que se ha seguido, estas se han asumido en relación con los objetivos estratégicos tanto de los sujetos particulares como del sistema en su conjunto.

De todo esto han surgido posibles escenarios y concretas hipótesis de intervención estratégica, que, precisamente, no se pueden separar de la realización de un diseño organizativo. El cual se basa en un modelo dividido en tres niveles diferentes, pero al mismo tiempo inseparables.

Primer nivel-Observatorios de cluster.

Son estructuras "abiertas" y flexibles, que implican la participación tanto de representantes de empresa como de organismos públicos y privados que comparten con los primeros problemas y objetivos (por lo demás establecidos a lo largo de los trabajos de análisis). Cada observatorio gira alrededor de la figura de un "especialista de cluster" al que se le atribuyen funciones de coordinación inicial de los sujetos participantes y de definición de las líneas de acción. El mismo coordinador interactúa con los demás coordinadores de observatorio, dialogando sobre la creación de proyectos y estrategias que impliquen a sistemas diferentes.

Las funciones de observatorio son, por lo tanto, las siguientes:

i) Reelaboración de los resultados cognoscitivos que han surgido de los análisis, estableciendo las profundizaciones necesarias;

ii) Determinación de los problemas y de las exigencias específicas del cluster al que se pertenece;

iii) Promoción de las hipótesis de trabajo a otros sujetos (empresas, instituciones) potencialmente interesados y promoción de su posible participación;

iv) Determinación y elaboración de hipótesis específicas de intervención estratégica.

Segundo nivel - Mesa de los actores institucionales.

Se compone de los representantes de las Administraciones municipales, provinciales y regionales (o sea los tres niveles político-administrativos que operan a nivel local), la Unión Industrial, la Asociación de Pequeñas Empresas, la Asociación artesana, la Cámara de Comercio, los Sindicatos de los trabajadores, la Universidad y el Politécnico de Turín y, sucesivamente, de los representantes de los Observatorios de cluster.

Recibiendo las sugerencias que provengan de los trabajos de observatorio, su función se centra en la definición de las condiciones y de las oportunidades políticas de realización de los proyectos definidos en el nivel precedente, valorando su nivel de coherencia con respecto a las líneas de acción estratégica ya en vías de ejecución.

Tercer nivel - Agencia para el desarrollo local.

Esta se constituye a través de la reasignación de personal dirigente y empleado que ya opera en los diferentes niveles institucionales aunque se ha previsto la contratación de personal ad hoc. 
Su función es doble:

1. Gestión de proyectos estratégicos de política industrial. De este modo, la Agencia se propone como nexo de unión entre observadores de cluster y empresas y otros organismos (promotores, instituciones de investigación, etc.) a los que se solicita la realización de los diferentes proyectos;

2. Gestión de los procesos de empresa establecidos. Esta afecta a los proyectos de simplificación de la normativa establecida; la aportación de un apoyo informativo adecuado a cada operador por un lado, y por otro, a los observatorios de cluster. En las primeras fases de realización se ha decidido además que la Agencia se ocupe de "compartir" los conocimientos generalizados presentes en el sistema local turinés.

Operativamente, la misma se compone de tres unidades diferentes:

1. Centro autónomo de elaboración de la información (con relación a aspectos ocupacionales, financieros y a los mercados y su distribución, etc.), de censo y recogida de las iniciativas de investigación ya en acto en instituciones públicas y privadas, académicas o no académicas. De este modo, se responde a la exigencia de búsqueda de una gestión integrada de la información (a través de la recogida y la introducción en la red de la información recogida, la gestión de sitios de Internet para uso de las empresas, la creación de bancos de datos específicos).

2. Separación de las oficinas urbanísticas de los ayuntamientos del área con el fin de realizar de modo coordinado las gestiones establecidas. Esta constituiría la única referencia para las empresas que pretenden establecerse, ampliarse, reubicarse. A ella le compete además la asistencia a las empresas a nivel de planificación logística y los complejos procesos dirigidos a la obtención y a la gestión de las financiaciones de la Unión Europea, los cuales, como ya se sabe, comportan la relación constante con una pluralidad de actores públicos y privados.

3. Centro de apoyo a las actividades de formación, de comercialización y de innovación. Responde a los problemas fundamentales planteados por las empresas entrevistadas, que han establecido, junto a específicos problemas de logística, la exigencia de tener a disposición un tejido conectivo que les consienta el acceso guiado a los proyectos y a los programas identificados como base de la competitividad del sistema manufacturero local.

Los tres proyectos estratégicos establecidos con anterioridad (el Parque del Diseño y de la creatividad, el Parque tecnológico y la hipótesis Building Automation) representan, de este modo, el banco de pruebas en el que se están comprobando las posibilidades de construir la ciudad del nuevo milenio.

\section{BIBLIOGRAFÍA.}

- ANTONELli, C. (1990), «L'economia fra mercato e organizzazione», in Bagnasco, A. (a cura di) La città dopo Ford. Il caso di Torino, Bollati Boringhieri, Torino, pp. 92-107.

- ASHEIM B.T. and ISAKSEN, A. (1997), «Location, innovation and agglomeration: towards regional innovation systems in Norway?», Europ. Plann. Studies, 5, 3, pp. 299-330.

- CEC (1994) Competitiveness and Cohesion: Trends in the Regions, Brus- 
sels.

- CONTI, S. and ENRIETTI, A. (1995), «The Italian automobile industry and the case of Fiat: one country, one company, one market?», in HUDSON, R. and SCHAMP, E.W. (eds), Towards a New Map of Automobile Manufacturing in Europe? New Production Concepts and Spatial Restructuring, Springer-Verlag, Berlin, pp. 117-46.

- COOKE P. and MORGAN, K. (1993), «The network paradigm: new departures in corporate and regional development», Environ. Plann. D, 11, pp. 54364.

- DE VET, J. (1993), «Globalisation and local and regional competitiveness», STI Revew, 13, OECD, Paris.

- DOERINGER, P. and TERKLA, D. (1990), «How intangible factors contribute to economic development», World Development, 18, 1, pp. 295-308.

- Dosi (1988) (et al.), Technical Change and Economic Theory, Pinter, London.

- DUNFORD, N. (1994), «Winners and losers: the new map of inequality in the European Union», Europ. Urban \& Reg. Studies, 1, 2, pp. 95-114.

- FosS, H.J. (1996), «Higher order industrial capabilities and competitive advantage», Journal of Industry Studies, 3, 1, pp. 1-20.

- FREEMAN, C. (1994), «Critical survey: the economics of technical change», Cambridge Journal of Economics, 18, pp. 463-512.

- GRANOVETTER, M. (1985), «Economic action and social structure. The problem of emdeddedness», American Journal of Sociology, 91, 3, pp. 481-510.

- HAAS, P.M. (1992), «Introduction: epistemic communities and international policy coordination», International Organisation, 46, pp. 1-35.

- Hudgson, G.M. (1993), Economics and Evolution: Bringing Life Back into Economics, Polity, Cambridge.

- HudSON, R. (1994), «Institutional change, cultural transformation, and economic regeneration: myths and realities from Europe's old industrial areas», en AMIN, A. and THIFT, N. (eds) Globalization, Institutions, and Regional Development in Europe, Oxford University Press, New York, pp. 196-216.

- LuNDVALL, B.A. (1992), «Introduction», en LuNDVALL, B.A. (ed.) National Systems of Innovation, Pinter, London, pp. 1-19.

- LUNDVALL, B.A. and JOHNSON, B. (1994), «The learning economy», Journal of International Studies, 1, 2, pp. 23-42.

- MORGAN, K. (1997), «The learning region: institutions, innovation and regional renewal», Regional Studies, 31, 5, pp. 491-504.

- OCDE (1991), Les rèsources technologiques et les stratégies de competitivité des petites et moyennes entreprises, et sur l'acces des PME au système de recherche (dr. S. CONTI), Direction de la Science, de la Tecnologie et de l'Industrie, Paris.

- Penrose, E. (1959), The theory of the Growth of the Firm, Basil Blackwell, Oxford.

- PiOre, M. and SABEL, C. (1994), The Second Industrial Divide, Basic Books, New York.

- PUTNAM, R. (1993), «The prosperour community: social capital and public 
life», American Prospec., 13, pp. 53-42.

- REHFELD, D. (1995), «Disintegration and reintegration of production clusters in the Ruhr area», en COOKE, P. (ed.), The Rise of the Rustbelt, University College London Press, London, pp. 85-102.

- SALAIS, R. and STORPER, M. (1993), Les mondes de production. Enquête sur l'identité économique de la France, Editions de l'école des hautes études en sciences sociales, Paris.

- Volpatto, G. (1996), Il caso Fiat, Isedi, Milano.

RESUMEN: Este artículo plantea el problema de la competitividad local/regional en la era de la globalización económica contextualizado en el ámbito del debate abierto en la ciencia geográfica y la ciencia social en general. Se presta especial atención en buscar un nexo claro con el fenómeno típico de la economía contemporánea, en la que se observa, de un lado, una creciente desindustrialización de la economía occidental, y de otro lado, la búsqueda paralela de actividad y actores dentro de su propio ámbito de referencia y acción.

Este cuadro dialéctico y contradictorio se ilustra de forma empírica con la referencia al caso de la región manufacturera de Turín, un área altamente industrializada que ha representado en el siglo que termina uno de los modelos más notables de organización fordista del trabajo, centrado en la producción automovilística. La transición hacia una economía post-fordista muestra en particular la ruptura con aquella lógica organizativa y la afirmación de nuevos sistemas productivos que reclaman, consiguientemente, nuevos modos de conocimiento y políticas.

PALABRAS CLAVE: Competitividad territorial, sistema, políticas de desarrollo local, sistema de valores, innovación, economía del conocimiento.

ABSTRACT: In this article, the problem of local/regional competitiveness in the era of the globalization of economic processes is first contextualised in the framework of the current debate in the geographical and social sciences in general. Particular care will be taken to seek out a link as clear as possible with the phenomena typical of the contemporary economy, in which we observe, on the one hand, the growing deindustrialisation of the Western economies and, on the other, a parallel rooting of activities and actors in their respective frameworks of reference and action.

This dialectical and contradictory framework will then be clarified empirically with reference to the case of Turin's manufacturing region, a highly industrialised area which in the century that has just finished represented one of the most well-known models of Fordist organisation of labour, centred on the car industry. The transition towards a post-Fordist economy shows in particular the profound break with those organisational approaches and the rise of new production clusters, and underlines the consequent need for new knowledge and policies.

KEY WORDS: Territorial competitiveness, clusters, local development policies, value systems, innovation, knowledge economy.

RESUMÉ: Cet article pose le problème de la compétitivité locale/régionale à l'ère de la globalisation économique contextualisé dans le cadre du débat ouvert de la science géographique et de la science sociale en général. On prête une attention spéciale à chercher un rapport clair avec le phénomène typique de l'économie contemporaine, où l'on observe, d'une part, une industrialisation croissante, et, d'autre part, la recherche parallèle d'activité et d'acteurs au sein de leur propre cadre de référence et d'action. 
Ce cadre dialectique et contradictoire s'illustre de façon empirique avec la référence au cas de la région manufacturée de Turin, une zone hautement industrialisée qui a représenté, durant le siècle qui s'achève, l'un des modèles les plus remarquables d'organisation fordiste du travail,centré sur la production automobile. La transition vers une économie post-fordiste.montre en particulier la rupture avec cette logique organisative et l'affirmation de nouveaux systèmes qui réclament, par conséquent, de nouvelles politiques ainsi que de nouveaux moyens de connaissances.

MOTS CLES: compétitivité territoriale, système, politiques de développement local, système de valeurs, innovation, économie de la connaissance. 